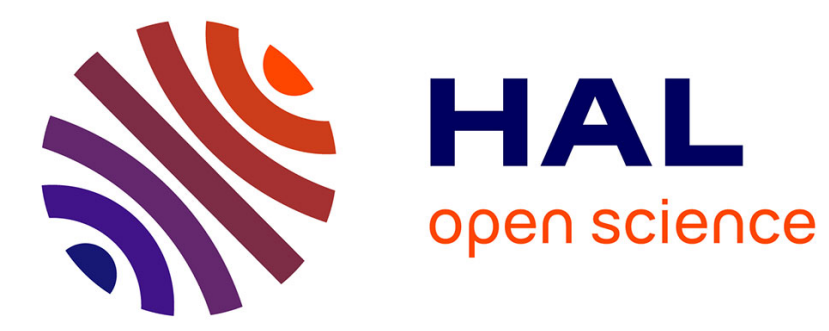

\title{
Variabilité génétique de la croissance musculaire et conséquences sur les qualités de la viande chez les bovins
}

\author{
G. Renand
}

\section{To cite this version:}

G. Renand. Variabilité génétique de la croissance musculaire et conséquences sur les qualités de la viande chez les bovins. Productions Animales, 1988, 1 (2), pp.115-121. hal-00895823

\section{HAL Id: hal-00895823 \\ https://hal.science/hal-00895823}

Submitted on 1 Jan 1988

HAL is a multi-disciplinary open access archive for the deposit and dissemination of scientific research documents, whether they are published or not. The documents may come from teaching and research institutions in France or abroad, or from public or private research centers.
L'archive ouverte pluridisciplinaire HAL, est destinée au dépôt et à la diffusion de documents scientifiques de niveau recherche, publiés ou non, émanant des établissements d'enseignement et de recherche français ou étrangers, des laboratoires publics ou privés. 
INRA Prod. Anim.

1988,1 (2), 115-121

\section{G. RENAND}

\section{INRA Jouy}

Station de Génétique

quantitative et appliquée

78350 Jouy-en-Josas

\section{Variabilité génétique de la croissance musculaire et conséquences sur les qualités de la viande chez les bovins.}

\begin{abstract}
Les aptitudes bouchères correspondent à l'ensemble des caractéristiques des bovins qui interviennent dans la transformation par l'animal de produits végétaux en viande consommable par l'homme. Or ce processus s'inscrit dans une filière qui fait intervenir de nombreux participants ayant chacun un objectif spécifique que ce soit l'éleveur-naisseur, l'engraisseur, l'abatteur, le transformateur, le boucher ou le consommateur.

Parmi toutes les caractéristiques de l'animal, le potentiel de croissance musculaire représente celle dont l'influence sur l'efficacité de la filière est la plus importante à trois niveaux. D'abord au niveau de l'éleveurnaisseur, du fait de ses relations avec la vitesse de croissance des veaux jusqu'au sevrage et le poids des vaches de réforme. Ensuite, au niveau de l'engraisseur qui, dans
\end{abstract}

\section{Résumé}

Parmi les aptitudes bouchères, la vitesse de croissance musculaire représente le principal objectif de sélection pour améliorer l'efficacité de la production de viande bovine au niveau de l'éleveur, de l'engraisseur et du boucher. A cette fin, il est possible d'utiliser les races à viande spécialisées qui possèdent simultanément une forte vitesse de croissance et une teneur en muscle élevée. Vu l'importante variabilité génétique intra-race (coefficients d'héritabilité élevés) il est également possible d'améliorer la croissance musculaire par sélection, si toutefois la composition corporelle des animaux peut être estimée correctement. Les qualités de la viande font partie des objectifs d'amélioration des seuls transformateur et consommateur et ne sont pas prises en considération, jusqu'à présent, dans les programmes d'amélioration génétique des aptitudes bouchères. Les études de comparaison de races ou les estimations d'héritabilité intra-race montrent qu'il existe une variabilité génétique non négligeable des qualités de la viande, bien que nettement plus faible que celle de la croissance musculaire. Parmi les différents critères de qualité, ceux liés à la tendreté expriment la plus grande variabilité génétique.

Les relations génétiques entre les qualités de la viande et la croissance musculaire estimées entre races ou intra-race ne permettent pas de conclure sur une possible modification de la tendreté lors d'une sélection sur la croissance musculaire. un système intensif, doit valoriser les aliments avec la meilleure efficacité alimentaire possible. Enfin au niveau de l'abatteur et du boucher qui recherchent des animaux avec les meilleurs rendements en viande possibles. Le potentiel de croissance musculaire est donc retenu comme principal objectif de sélection pour améliorer les aptitudes bouchères.

Par contre, au niveau du transformateur ou du consommateur, l'incidence d'une telle sélection sur les qualités de la viande est très mal connue. Jusqu'à présent ces qualités de la viande n'ont pu être intégrées dans les objectifs de sélection faute de méthodes de mesure simples et faute d'une évaluation de leur impact économique.

\section{1 / La variabilité génétique de la croissance musculaire}

La croissance musculaire s'apprécie à travers la vitesse de croissance et la composition corporelle qui est mesurable à l'abattage par dissection de tout ou partie de la carcasse. Dans la littérature, il existe de nombreuses estimations des différences entre races, ainsi que des paramètres génétiques intra-race pour ces caractéristiques.

\section{1 / Variation entre races}

Entre races, il apparait de très importantes différences de croissance musculaire (tableau 1) avec, à un extrême, des races à viande spécialisées ou des races ou lignées culardes (Charolaise, Blonde d'Aquitaine, Blanc Bleu Belge, Piémontaise et INRA 95) et, à l'autre, des races comme la Jersiaise ou l'Angus dont la croissance musculaire est inférieure de $20 \%$ à celle des précédentes. 
Tableau 1. Effets de diverses races paternelles sur différentes aptitudes bouchères : déviations absolues $(\Delta)$ ou relatives $(\%)$ à la race paternelle Charolaise.

(Synthèse de résultats bibliographiques, Renand et al à paraître).

\section{En production \\ de viande bovine, \\ la croissance \\ musculaire représente \\ un objectif de sélection primordial.}

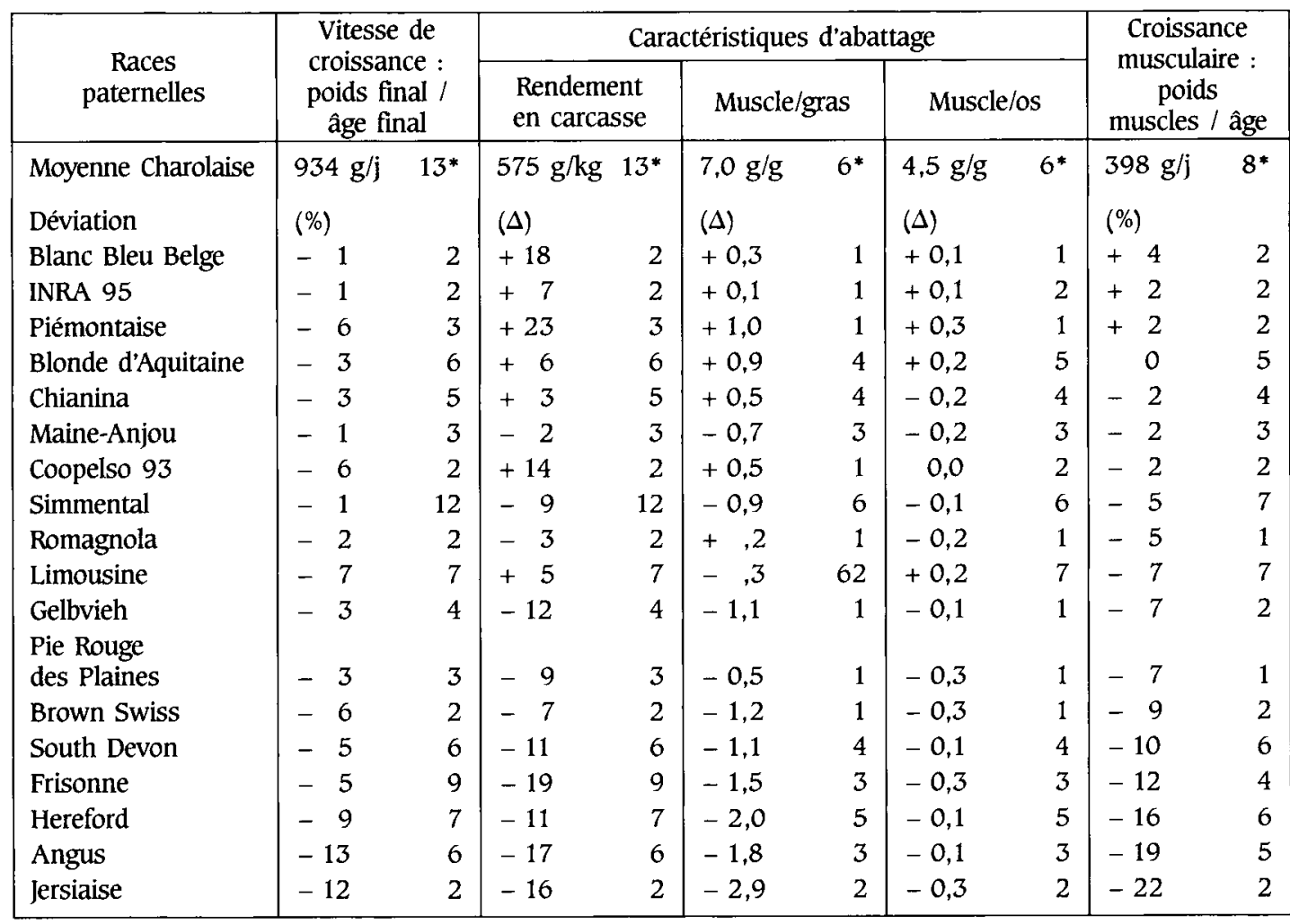

* : nombre d'expériences.

Cette croissance musculaire est fortement liée à la vitesse de croissance en vif et donc au poids adulte. Mais il faut également noter que des races de plus petite taille peuvent avoir une croissance musculaire identique, voire supérieure, à des races de grande taille grâce à une composition corporelle nettement plus favorable. Ceci est le cas de la race Limousine vis-à-vis de la race Simmental par exemple.

\section{2 / Héritabilités}

Intra-race, il existe également une importante variabilité génétique des aptitudes bouchères dépendant de la croissance musculaire : la vitesse de croissance pendant l'engraissement et la composition corporelle à l'abattage. Cette variabilité s'apprécie à travers l'héritabilité de ces caractères (tableau 2). Avec des coefficients d'héritabilité voisins de 0,50 il apparaît donc facile théoriquement de modifier par sélection le niveau de crois- sance musculaire d'une population. La réalisation d'une telle amélioration se heurte aux problèmes techniques d'estimation de la composition corporelle. A l'heure actuelle, seul l'abattage et une dissection partielle en permettent une estimation suffisamment précise. On ne peut donc sélectionner les taureaux qu'après un contrôle sur descendance ce qui allonge l'intervalle de génération et surtout limite le nombre de taureaux à évaluer et donc réduit l'intensité de sélection.

Une phase de sélection sur performances individuelles des taureaux est absolument nécessaire pour assurer un minimum de progrès génétique. Faute d'estimateurs précis de la composition corporelle, seule la vitesse de croissance peut être sélectionnée. Or, la vitesse de croissance est la résultante de deux caractéristiques des animaux : leur capacité d'ingestion et leur efficacité alimentaire. Sachant d'abord que l'énergie ingérée en sus des besoins d'entretien et de croissance protéique (dont la moitié se réalise au niveau des muscles) est stockée

Tableau 2. Coefficient d'héritabilité $\left(h^{2}\right)$ des aptitudes bouchères liées à la croissance musculaire (synthèse de résultats bibliographiques, Renand et al à paraître).

\begin{tabular}{|l|c|c|}
\hline \multicolumn{1}{|c|}{ Caractère } & $\begin{array}{c}\text { Héritabilité } \\
\text { moyenne }\end{array}$ & $\begin{array}{c}\text { Nombre } \\
\text { d'estimées }\end{array}$ \\
\hline GMQ post-sevrage & 0,46 & 48 \\
Poids fin d'engraissement & 0,52 & 16 \\
Rendement à l'abattage & 0,47 & 26 \\
Poids de muscle & 0,56 & 12 \\
Poids de gras & 0,66 & 11 \\
Teneur en muscle & 0,44 & 31 \\
Teneur en gras & 0,49 & 27 \\
\hline
\end{tabular}


Tableau 3. Coefficients de corrélations génétiques (rg) entre performances d'engraissement et d'abattage (synthèse de résultats bibliographiques, Renand et al à paraître).

\begin{tabular}{|l|c|c|c|c|c|}
\hline \multicolumn{1}{|c|}{ Caractère } & GMQ & $\begin{array}{c}\text { Poids } \\
\text { de muscle }\end{array}$ & $\begin{array}{c}\text { Poids } \\
\text { de gras }\end{array}$ & $\begin{array}{c}\text { Teneur } \\
\text { en muscle }\end{array}$ & $\begin{array}{c}\text { Teneur } \\
\text { en gras }\end{array}$ \\
\hline Consommation journalière & $+0,81$ & $+0,72$ & $+0,68$ & $-0,17$ & $+0,24$ \\
GMQ post-sevrage & 1,00 & $+0,85$ & $+0,45$ & $+0,03$ & $-0,15$ \\
Efficacité alimentaire & $+0,52$ & $+1,07$ & $+0,03$ & $+0,22$ & $-0,28$ \\
\hline
\end{tabular}

dans les dépôts adipeux et ensuite que le coût énergétique de la croissance adipeuse est nettement plus élevé que celui de la croissance musculaire, les hypothèses suivantes peuvent être avancées. Si l'amélioration de cette vitesse de croissance est essentiellement liée à une amélioration de l'efficacité alimentaire il est probable que les animaux sélectionnés auront un meilleur potentiel de croissance musculaire. Par contre si l'amélioration de la vitesse de croissance est surtout le fait d'une augmentation de la capacité d'ingestion il n'est pas sûr que le potentiel de croissance musculaire soit amélioré car les dépôts adipeux des animaux seront plus importants et plus précoces. Ces hypothèses sont confirmées, entre autres, par les quelques paramètres génétiques qui ont été obtenus simultanément sur la vitesse de croissance, les quantités d'aliment consommées, l'efficacité alimentaire et la composition des carcasses (tableau 3). Pour cette raison, les taureaux de races à viande destinés à l'insémination artificielle en France sont sélectionnés sur leurs performances individuelles de croissance et d'efficacité alimentaire contrôlées en station (Ménissier et al 1986).

\section{2 / La variabilité génétique des qualités de la viande et les relations avec la croissance musculaire}

Il n'existe dans la littérature que très peu d'estimations de la variabilité génétique entre races ou intra-race pour les qualités de la viande.

\section{1 / Variation entre races}

Il est possible de citer trois expériences de croisement terminal effectuées avec diverses races paternelles utilisées soit sur des femelles allaitantes Hereford-Angus au Nebraska (Gregory et al 1982), soit sur des femelles de races laitières au Danemark (Liboriussen et al 1982) ou en France (Bonaiti 1980, Ménissier et al 1982). Les résultats de ces expériences sont synthétisés par les figures 1 à 3 . Pour chaque étude, une analyse en composantes principales a été réalisée sur les critères de qualité de la viande et deux figures sont présentées. Sur la première sont reportées les corrélations entre les diverses caractéristiques de qualité et les deux premières composantes principales. Sur cette même figure sont reportées les corrélations entre, d'une part, ces composantes principales et, d'autre part, la croissance musculaire (muscle/jour de vie) et la teneur en gras de la carcasse. Sur la seconde sont reportées la position des différentes races paternelles relativement à ces composantes principales.

De l'ensemble de ces études, il ressort que pour les qualités de la viande, le différences entre races ne sont généralement pas significatives compte tenu de la forte variabilité individuelle observée. Les seules différences qui soient réellement significatives concernent la moindre tendreté de la viande des animaux issus de taureaux zébus comparativement aux autres types génétiques dans l'expérience du Nebraska et des animaux issus de taureaux Frisons dans l'expérience danoise.

L'intérêt de ces études n'est pas tant de montrer qu'il existe relativement peu de différences significatives entre races mais plutôt de permettre de mieux appréhender les relations inter-raciales entre les divers critères de qualité de la viande et entre ceux-ci et la croissance musculaire.

Au Nebraska (figure 1) les qualités organoleptiques de la viande (tendreté, jutosité, flaveur) ont été jugées par un jury de dégustation. Une mesure physique de la dureté (force de cisaillement) a été réalisée et une estimation subjective de l'importance des dépôts adipeux intramusculaire a été faite à travers la note de persillé.

Figure 1. Analyse en composantes principales des qualités de la viande: variabilité entre races. Nébraska (Gregory et al 1982).


Par contre,

les qualités

de la viande sont moins variables et difficiles à prendre en compte comme critères de sélection. 
Il apparaît qu'une part importante de la variabilité entre races est expliquée par la première composante principale $(75 \%)$ qui oppose les trois critères de dégustation à la force de cisaillement. La seconde composante n'explique que $12 \%$ de la variabilité et discrimine les races sur leur note de persillé. Cette dernière est toutefois assez fortement corrélée $(+0,7)$ avec la première composante. C'est-à-dire que les races dont la viande est tendre et jugée satisfaisante sont des races qui possèdent également une note de persillé relativement élevée.

Parmi les critères de qualité de la viande la note de persillé est très étroitement liée avec la teneur en gras de la carcasse et donc fortement opposée avec la croissance musculaire. De ce fait, il apparaît une relation entre races légèrement défavorable $(-0,2)$ entre la croissance musculaire et la première composante principale des qualités de la viande du fait que les races les plus grasses ont des viandes mieux appréciées que les races maigres.

Au Danemark (figure 2), des qualités organoleptiques furent également jugées par un jury de dégustation (acceptabilité et tendreté) et la dureté mesurée par une force de cisaillement. En plus, un dosage de la teneur en lipides intramusculaires a été fait, de même qu'une mesure de réflectance sur le muscle long dorsal. La première composante (44\%) explique l'essentiel des différences raciales de tendreté et d'acceptabilité de la viande, et elle est semblable à la première composante mise en évidence dans l'expérience précédente. La deuxième composante $(22 \%)$ explique essentiellement les diffé-

Figure 2. Analyse en composantes principales des qualités de la viande : variabilité entre races. Danemark (Liboriussen et al 1982).



rences de teneurs en lipides intramusculaires indépendamment du premier critère de qualité. Sur cet axe, il apparaît que les races dont la viande contient le plus de lipides ont tendance à avoir des viandes plus sombres. En fait, ce critère de couleur est plutôt indépendant des autres variables de qualité de la viande et explique à lui seul la troisième composante principale.

Alors que la teneur en lipides intramusculaires est indépendante de la première composante, la teneur en gras de la carcasse, qui lui est étroitement liée, présente une légère opposition avec cette première composante, contrairement à ce qui a été observé dans l'expérience précédente. De ce fait, la liaison entre croissance musculaire et qualités de la viande (tendreté, acceptabilité) est plutôt favorable entre les races étudiées $(+0,4)$.

En France (figure 3), les qualités de la viande ont été étudiées à travers des mesures physico-chimiques sans faire l'objet de dégustation. Les mesures sont la force de cisaillement, le $\mathrm{pH}$, la réflectance, la perte en eau,

Figure 3. Analyse en composantes principales des qualités de la viande: variabilité entre races. France (Bonaiti 1980, Ménissier et al 1982).

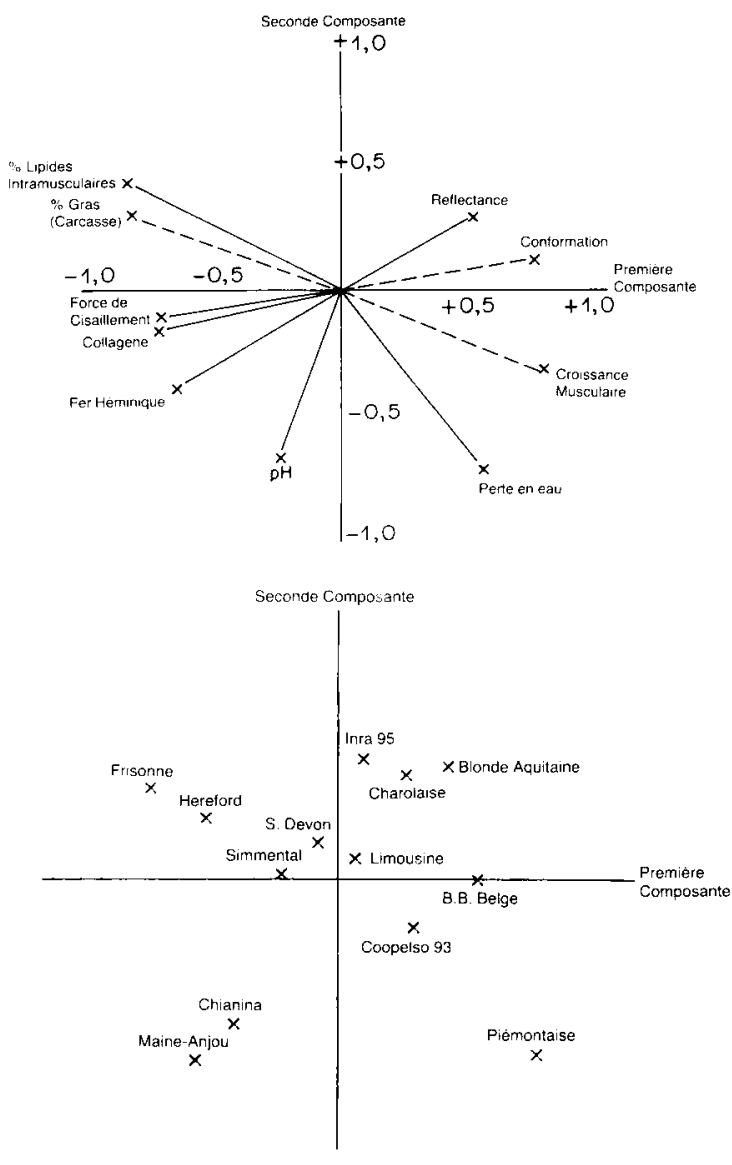

et les teneurs en collagène, en lipides intramusculaires et en fer héminique. L'analyse en composantes principales montre que la variabilité entre les races étudiées reflète tout d'abord une variabilité conjointe de la teneur en gras intramusculaire et de la dureté ( $40 \%$ ). La seconde composante $(21 \%)$ fait plutôt intervenir la variabilité qui existe pour le $\mathrm{pH}$ et le pouvoir de rétention d'eau. Il faut noter tout d'abord que les différences 
Tableau 4. Coefficients d'héritabilité des critères de qualité de la viande

(synthèse de résultats bibliographiques : Gravert 1962a et b, Dubose et Cartwright 1967, Wilson et al 1971, Dinkel et Busch 1973, Wilson et al 1976, Andersen et al 1977, Ender 1981, Koch et al 1982, Renand 1985, Oldenbroeck et Meijering 1986).

\begin{tabular}{|l|c|c|}
\hline \multicolumn{1}{|c|}{ Caractère } & $\begin{array}{c}\text { Héritabilité } \\
\text { moyenne }\end{array}$ & $\begin{array}{c}\text { Nombre } \\
\text { d'estimées }\end{array}$ \\
\hline Note d'acceptabilité & 0,30 & 1 \\
Note de tendreté & 0,26 & 3 \\
Note de jutosité & 0,11 & 3 \\
Note de flaveur & 0,08 & 3 \\
Force de cisaillement & 0,33 & 8 \\
Couleur (réflectance) & 0,29 & 8 \\
pH final & 0,28 & 4 \\
Perte en eau & 0,20 & 6 \\
Teneur en gras intramusculaire & 0,26 & 3 \\
Teneur en eau du muscle & 0,20 & 1 \\
Teneur en hydroxyproline & 0,00 & 1 \\
Diamètre moyen des fibres & 0,34 & 2 \\
\% fibres rouges & 0,24 & 1 \\
\% fibres blanches & 0,22 & 1 \\
\hline
\end{tabular}

raciales de teneur en collagène et de force de cisaillement sont fortement liées et donc que l'une quelconque de ces deux mesures peut être utilisée pour apprécier la dureté de la viande. Mais, surtout, il apparaît que les races donnant la viande la plus riche en lipides sont également les races dont la viande est la plus dure. Et comme la teneur en lipides intramusculaires est fortement liée à l'état d'engraissement des carcasses, il apparaît une relation nettement favorable entre croissance musculaire et tendreté $(+0,7)$ encore plus marquée que dans l'expérience danoise. Les races à forte croissance musculaire apparaissent également comme ayant des viandes plus claires (Blonde d'Aquitaine, Charolaise) avec une moindre capacité de rétention de l'eau (Piémontaise).

De ces comparaisons des qualités de la viande entre races il apparaît dans tous les cas que le principal facteur de discrimination est la tendreté et que les appréciations des jurys de dégustation dépendent fortement de celle-ci. Par contre, il existe de nettes différences entre ces trois expériences sur la relation qu'il peut exister entre cette tendreté et la teneur en lipides de la viande : relation positive au Nébraska, nulle au Danemark et nettement négative en France. Or, comme les races qui déposent le plus de lipides intra-musculaires sont égalemnet celles dont les dépôt adipeux de la carcasse sont les plus importants $(r=+0,9)$ et dont la croissance musculaire est la plus faible $(r=-0,8)$, on observe des différences entre expériences sur la relation qui apparaît entre tendreté et croissance musculaire. Au Nébraska, où les mesures ont été effectuées sur des mâles castrés, la relation est légèrement défavorable. En Europe, sur des taurillons, cette relation est favorable, surtout en France. Ces différences doivent trouver leurs origines dans le choix des races, des critères de qualités de la viande et des conditions d'engraissement et d'abattage.

\section{2 / Héritabilités}

Il est possible de trouver quelques estimations des paramètres génétiques mais beaucoup moins nombreuses que pour les performances d'engraissement ou d'abattage. Il faut donc interpréter avec prudence les estimations moyennes rapportées dans le tableau 4. Il apparaît que pour les mesures physiques qui ont été le plus souvent réalisées (force de cisaillement, réflectance, $\mathrm{pH}$, perte en eau), les coefficients d'héritabilité sont inférieurs aux coefficients estimés pour les aptitudes bouchères liées à la croissance musculaire. Ces coefficients se situent entre 0,20 et 0,30 en moyenne. Ils indiquent toutefois l'existence d'une variabilité génétique additive non négligeable qui peut être exploitée par sélection si tel est l'objectif. Pour les autres critères de qualité de la viande le faible nombre d'estimées de l'héritabilité ne permet pas de conclure sur les possibilités de pratiquer une sélection efficace, mais on peut noter que les coefficients d'héritabilité ne dépassent pas 0,30 en général.

\section{3 / Corrélations génétiques}

L'existence d'une variabilité génétique certaine mais également l'absence d'une sélection directe sur ces caractères conduisent à s'intéresser aux corrélations génétiques qui détermineront l'évolution des qualités de la viande lors d'une sélection sur les aptitudes bouchères (croissance musculaire).

Tout d'abord une étude française a montré qu'il n'existe pratiquement aucune relation génétique entre les trois critères suivants : force de cisaillement, $\mathrm{pH}$ et perte en eau (tableau 5). Une étude danoise a confirmée qu'intrarace il existe, comme entre races, une étroite relation génétique entre les différentes qualités organoleptiques jugées par un jury de dégustation (acceptabilité, tendreté, flaveur, jutosité) et entre ces dernières et la dureté appréciée par une force de cisaillement (figure 4). Ces caractéristiques sont liées à une moindre capacité de rétention de l'eau mais sont indépendantes de la teneur en lipides intramusculaires. Dans cette étude, il apparaît aussi une opposition génétique entre la couleur de la viande et la teneur en lipides intramusculaires.

Tableau 5. Coefficients de corrélations génétiques entre critères de qualité de la viande (Renand 1985).

\begin{tabular}{|l|c|c|}
\hline & $\begin{array}{c}\text { pH } \\
\text { final }\end{array}$ & $\begin{array}{c}\text { Perte } \\
\text { en eau }\end{array}$ \\
\hline Force & & \\
de cisaillement & $-0,03$ & $-0,06$ \\
pH final & 1,00 & $-0,13$ \\
\hline
\end{tabular}

\section{II n'est pas possible actuellement de chiffrer l'incidence de la sélection sur la tendreté, principale composante des qualités de la viande.}


Figure 4. Analyse en composantes principales des qualités de la viande: corrélations génétiques intra-race (Andersen et al 1977).



Enfin, pour appréhender les conséquences sur les qualités de la viande d'une sélection sur la croissance musculaire, il existe très peu de données bibliographiques. Les quelques coefficients de corrélations génétiques publiés jusqu'à présent sont rapportés dans le tableau 6 . Il semblerait qu'une sélection visant à améliorer la croissance musculaire ait pour conséquence une couleur plus claire de la viande, une augmentation $\mathrm{du} \mathrm{pH}$ et surtout une réduction de la teneur en lipides intramusculaires. Par contre, pour ce qui concerne la tendreté il n'est pas possible de tirer des conclusions puisque deux études indiquent une détérioration possible de celle-ci lors d'une sélection pour une forte croissance musculaire alors qu'un troisième indiquerait plutôt une très légère amélioration.

\section{Conclusion}

Il existe une variabilité génétique importante de la croissance musculaire chez les bovins. Il est donc possible de l'améliorer en exploitant les différences entre races ou en sélectionnant les reproducteurs intra-race. Par contre, la variabilité génétique des qualités de la viande est moins marquée aussi bien entre races qu'intra-race. Elle est plus particulièrement appréciable pour les critères relatiffs à la dureté qui constitue la principale composante génétique des qualités de la viande.

L'information actuellement disponible sur les relations génétiques intra-race comme sur les relations entre races montre qu'une amélioration de la croissance musculaire va de pair avec une réduction de la teneur en lipides du muscle, un éclaircissement de la couleur de la viande et une tendance vers une moins bonne rétention de l'eau. Par contre, il ne nous est pas possible de prédire l'évolution de la principale composante des qualités de la viande : la tendreté, du fait de l'incohérence des diverses relations estimées aussi bien entre races qu'intrarace. Des études génétiques sont encore nécessaires pour comprendre l'influence sur cette relation de la variabilité de la teneur en lipides intramusculaires mais, certainement aussi, celle du degré de maturité des animaux.

Cet article reprend les résultats présentés lors de la réunion de la Commission des Recherches Bovines de I'INRA les 2 et 3 juin 1987 sur le thème "Croissance musculaire et qualités des viandes".

\section{Références bibliographiques}

ANDERSEN B.B., LYKKE TH., KOUSGAARD KR., BUCHTER L. WLMSER-PEDERSEN I., 1977. Growth, feed utilization, carcass quality and meat quality in Danish dual-purpose cattle. Beretn. fra Statens Husdryrbrugsfors, 453, $86 \mathrm{pp}$

BONAITI B., 1980. Influence du type génétique sur les caractéristiques et l'utilisation technologique de la carcasse de jeune bovin. Compte rendu d'une recherche financée par la D.G.R.S.T. $\mathrm{n}^{\circ} 78.7 .0467 \& 78.7 .0468$

DINKEL C.A., BUSCH D.A., 1973. Genetic parameters among production, carcass composition and carcass quality traits of beef cattle. l. Anim. Sci., 36, 832-846.

Tableau 6. Coefficients de corrélations génétiques entre les critères de qualité de la viande et les critères de croissance musculaire

\begin{tabular}{|c|c|c|c|c|c|c|c|c|c|}
\hline & & $\begin{array}{c}\text { Force de } \\
\text { cisail- } \\
\text { lement }\end{array}$ & $\begin{array}{l}\text { Note de } \\
\text { tendreté }\end{array}$ & $\begin{array}{l}\text { Réflec- } \\
\text { tance }\end{array}$ & $\begin{array}{l}\mathrm{pH} \\
\text { final }\end{array}$ & $\begin{array}{c}\text { Perte } \\
\text { en eau }\end{array}$ & $\begin{array}{c}\% \text { lipides } \\
\text { intra- } \\
\text { musculaires }\end{array}$ & $\begin{array}{c}\% \text { eau } \\
\text { du } \\
\text { muscle }\end{array}$ & $\begin{array}{l}\text { Diamètre } \\
\text { moyen } \\
\text { des fibres }\end{array}$ \\
\hline A & $\begin{array}{l}\text { GMQ } \\
\% \text { gras } \\
\text { carcasse }\end{array}$ & $\begin{array}{r}0,57 \\
-0,29\end{array}$ & $\begin{array}{r}-0,22 \\
0,32\end{array}$ & & & & & & \\
\hline B & $\begin{array}{l}\text { GMQ } \\
\% \text { gras } \\
\text { carcasse }\end{array}$ & $\begin{array}{r}0,43 \\
-0,31\end{array}$ & $\begin{array}{r}-0,40 \\
0,19\end{array}$ & $\begin{array}{r}0,23 \\
-0,30\end{array}$ & & & $\begin{array}{r}-0,91 \\
0,74\end{array}$ & & $\begin{array}{r}-0,07 \\
0,12\end{array}$ \\
\hline C & $\begin{array}{l}\text { GMQ } \\
\% \text { gras } \\
\text { carcasse }\end{array}$ & & & $\begin{array}{r}0,24 \\
-0,12\end{array}$ & $\begin{array}{r}0,15 \\
-0,22\end{array}$ & & & $\begin{array}{r}0,12 \\
-0,12\end{array}$ & \\
\hline D & $\begin{array}{l}\text { GMQ } \\
\% \text { gras } \\
\text { carcasse }\end{array}$ & $\begin{array}{l}0,01 \\
0,21\end{array}$ & & & $\begin{array}{r}0,21 \\
-0,51\end{array}$ & $\begin{array}{r}0,18 \\
-0,35\end{array}$ & & & \\
\hline & $\begin{array}{l}\text { ace } \\
\text { tire }\end{array}$ & $+?$ & - & + & + & + & - & + & - \\
\hline
\end{tabular}

A : Wilson et al 1976 (U.S.A.).

B : Andersen et al 1977 (Danemark)

C : Ender 1981 (R.D.A.)

D : Renand 1985 (France) 
DUBOSE L.E., CARTHWRIGHT T.C., 1973. Relationships amon production and carcass traits in cattle. J. Anim. Sci., 26, 203 (Abstr.).

ENDER K., 1981. Genetische Aspekte zur Fleishbeschaffenheit beim Rind. Tierzucht, 35, 207-210.

GRAVERT H.O., 1962a. Untersuchungen über die Erblichkeit von Fleischeigenschaften beim Rind. 1 : Fläche des Musc. Long. dorsi, Wassergehalt, Wasserverbindung, Fettgehalt. Z. Tierz. Züchtungsbiol, 78, 43-74.

GRAVERT H.O., 1962b. Untersuchungen über die Erblichkeit von Fleischeigenschaften beim rind, 2 : Eiweissgehalt, pH.wert, Farbe Muskelfaserstarke, Zartheit. Z. Tierz. Züchtungsbiol, 78, 139-178. GREGORY K.E., CUNDIFF L.V., KOCH R.M., 1982. Comparison in crossbreeding systems and breeding stocks used in suckling herds of continental and temperate areas. $2^{\mathrm{e}}$ Congr. Mond. Génét. Appl. Prod. Anim., Madrid, Espagne, 5: 482-503.

KOCH R.R., CUNDIFF L.V., GREGORY K.E., 1982. Heritabilities and genetic, environmental and phenotypic correlations of carcass traits in a population of diverse biological types and their implications in selection programs. J. Anim. Sci., 47, 142-150.

LIBORIUSSEN T., LAURITZEN F., ANDERSEN B.B., BUCHTER L. SORENSEN S.E., KLASTRUP S., KOUSGAARD K., 1982. Résultats de croisement avec des races à viande européennes : I et II. (en Danois). Beretn. fra Statens Husdyrbrugs fors., 527:65 pp.
MÉNISSIER F., SAPA J., FOULLEY J.L., FREBLING I., BONAITI B., 1982. Comparison of different sire breeds crossed with Friesian cows : preliminary results. Current Topics Vet. Med. Anim. Sci., 21, 94-136.

MÉNISSIER F., RENAND G., COLLEAU J.J., GAILLARD J., 1986. Amélioration génétique de la production de viande bovine : orientations, objectifs et méthodes de sélection des aptitudes bouchères. In : D. Micol éd., Production de viande bovine, 101-106, INRA, Paris.

OLDENBROECK ].K., MEIIERING A., 1986. Breeding for veal production in black and white dairy cattle. Livest. Prod. Sci., 15. 325-336.

RENAND G., 1985. Genetic parameters of French beef breeds used in crossbreeding for young bull production. II. Slaughter performance. Génét. Sél. Evol., 17, 265-282.

WILSON L.L., STOUT J.M., ZIEGLER J.H., SIMPSON M.J., VARELAALVAREZ H., RUGH M.C., WATKINS I.L., 1971. Heritability of live and carcass characters in a crossbred beef herd. J. Heredity, 62 123-125.

WILSON L.L., MC CURLEY J.R., ZIEGLER J.H., WATKINS J.L., 1976. Genetic parameters of live and carcass characters from progeny of polled Hereford sires and Angus-Holstein cows. J. Anim. Sci., 43, 569-576. 